\title{
Compositionally universal entire functions on the plane and the punctured plane
}

\author{
Luis Bernal-González \\ To Professor Tomás Dominguez Benavides on his 60th birthday
}

\begin{abstract}
Necessary/sufficient conditions for a sequence of automorphisms of the complex plane to generate a sequence of composition operators that is universal on the punctured plane are provided. As a consequence, it is derived that only for translations and rotation-dilations there can be entire functions whose orbits present universality. Boundedness of these functions on unbounded sets as well as frequent universality on the whole plane are also analyzed.
\end{abstract}

Mathematics Subject Classification (2010). Primary 30E10; Secondary 47A16, $47 \mathrm{~B} 33$.

Keywords. Universal entire functions, punctured plane, universal sequence of operators, hypercyclic functions, boundedness, frequent universality.

\section{Introduction, notation, known results and aim of this paper}

In 1929 Birkhoff [9] published his celebrated universality theorem asserting the existence of an entire function $f$ on the complex plane $\mathbb{C}$ whose translates $f(\cdot+n)$ $(n \in \mathbb{N}:=\{1,2, \ldots\})$ approximate uniformly any prescribed entire function on every compact subset of $\mathbb{C}$. His result can be put into the more general setting of universality, whose starting notions are collected in the next paragraph.

Assume that $X$ and $Y$ are topological spaces and that $T_{n}: X \rightarrow Y(n \geq 1)$ is a sequence of continuous mappings. Then $\left(T_{n}\right)$ is said to be universal whenever there exists a point $x_{0} \in X$, called universal for $\left(T_{n}\right)$, whose orbit $\left\{T_{n} x_{0}: n \geq 1\right\}$ under $\left(T_{n}\right)$ is dense in $Y$. The set of universal points for $\left(T_{n}\right)$ will be denoted by

The author has been partially supported by the Plan Andaluz de Investigación de la Junta de Andalucía FQM-127, by MCI Grant MTM2009-10696-C02-01 and by MCYT (Acción Especial) MTM2007-30904-E. 
$\mathcal{U}\left(\left(T_{n}\right)\right)$. It is easy to see that, if $X$ is a Baire space and $Y$ is second countable, then $\mathcal{U}\left(\left(T_{n}\right)\right)$ is dense if and only if it is residual, and if and only if $\left(T_{n}\right)$ is transitive (i.e. given nonempty open sets $U \subset X, V \subset Y$, there is $n \in \mathbb{N}$ such that $T_{n}(U) \cap$ $V \neq \emptyset$ ). If $X=Y$, then a single continuous selfmapping $T: X \rightarrow X$ is called universal (transitive) if the sequence $\left(T^{n}\right)$ of its iterates is universal (transitive, resp.), and we denote $\mathcal{U}(T)=\{$ universal points for $T\}:=\mathcal{U}\left(\left(T^{n}\right)\right)$. Note that $\mathcal{U}(T) \neq \emptyset$ if and only if $\mathcal{U}(T)$ is dense. If now $X$ and $Y$ are (Hausdorff) topological vector spaces and $\left\{T_{n}: n \in \mathbb{N}\right\} \subset L(X, Y)=:$ continuous linear mappings $X \rightarrow Y\}$ then the words universal and hypercyclic are synonymous, although "hypercyclic" is mainly used for single mappings $T \in L(X)=$ \{operators on $X\}:=L(X, X)$. The reader is referred to the surveys [4], [18] and [20] for history, concepts, results and references about universality and hypercyclicity.

For a domain (nonempty connected open subset) $G \subset \mathbb{C}$, we denote by $H(G)$ the vector space of all holomorphic functions $G \rightarrow \mathbb{C}$. Then $H(G)$ becomes an Fspace (that is, a complete metrizable topological vector space) when it is endowed with the topology of convergence on compacta; in addition, $H(G)$ is separable, hence second countable. We will denote by $\mathcal{M}(G)$ the family of Mergelyan subsets of $G$, that is, $\mathcal{M}(G)=\{K \subset \mathbb{C}: K$ is compact and $\mathbb{C} \backslash K$ is connected $\}$. Recall that the sets $U(f, \varepsilon, K):=\{g \in H(G):|g(z)-f(z)|<\varepsilon \forall z \in K\}(f \in H(G), \varepsilon>$ $0, K \subset G$ a compact set) form a basis of open subsets in $H(G)$. For the sake of brevity, we introduce the following two concepts. A subset $\mathcal{A} \subset H(G)$ will be called $\mathcal{M}$-dense provided that for every $\varepsilon>0$ and every $K \in \mathcal{M}(G)$, one has $\mathcal{A} \cap U(f, \varepsilon, K) \neq \emptyset$. By using Runge's approximation theorem (see [16]), we have that the polynomials form an $\mathcal{M}$-dense subset of $H(G)$. Of course, denseness matches $\mathcal{M}$-denseness if $G$ is simply connected. If $G, \Omega$ are domains in $\mathbb{C}$ and $\varphi \in H(G, \Omega)$ (i.e. $\varphi \in H(G)$ and $\varphi(G) \subset \Omega$ ), then the composition operator $C_{\varphi}: H(\Omega) \rightarrow H(G)$ is the continuous linear mapping defined as $C_{\varphi} f=f \circ \varphi$. If $\left(\varphi_{n}\right) \subset H(G, \Omega)$, then we say that the sequence of composition operators $\left(C_{\varphi_{n}}\right)$ is $\mathcal{M}$-universal provided that there is $f \in H(G)$-called $\mathcal{M}$-universal for $\left(C_{\varphi_{n}}\right)$-such that $\left\{f \circ \varphi_{n}\right\}_{n \geq 1}$ is $\mathcal{M}$-dense in $H(G)$. A corresponding notion of $\mathcal{M}$-hypercyclicity arises when $G=\Omega$ and each $\varphi_{n}$ is the $n$ th-iterate $\varphi^{[n]}=\varphi \circ \cdots \circ \varphi$ (n-fold) of $\varphi$.

In the terminology of the preceding paragraphs, Birkhoff's theorem tells us that the translation operator $f \mapsto f(\cdot+1)$ is hypercyclic on $H(\mathbb{C})$. With essentially the same proof as in [9] we have (see [22]) that if $\left(a_{n}\right) \subset \mathbb{C}$ is an unbounded sequence then the sequence of translations $\tau_{a_{n}}: f \in H(\mathbb{C}) \mapsto f\left(\cdot+a_{n}\right) \in H(\mathbb{C})$ $\left(n \in \mathbb{N}\right.$ ) is universal (a function $f \in H(\mathbb{C})\left(\tau_{a_{n}}\right)$-universal for some $\left(a_{n}\right) \subset \mathbb{C}$ will be called Birkhoff-universal). In 1988, Zappa [28] replaced the additive group $(\mathbb{C},+)$ by the multiplicative group $\left(\mathbb{C}^{*}:=\mathbb{C} \backslash\{0\}, \cdot\right)$ (see [1] and [2] for extensions to complex special or general linear groups) and demonstrated the existence of a "multiplicatively universal" function $f \in H\left(\mathbb{C}^{*}\right)$, that is, given $g \in H\left(\mathbb{C}^{*}\right), \varepsilon>0$ and $K \in \mathcal{M}\left(\mathbb{C}^{*}\right)$, there exists $a \in \mathbb{C}^{*}$ such that $|f(a z)-g(z)|<\varepsilon$ for all $z \in K$ (by Mergelyan's approximation theorem [16, Chap. 3], one may in fact prescribe $g \in A(K)$, the space of continuous functions $f: K \rightarrow \mathbb{C}$ that are holomorphic 
in the interior $K^{0}$ of $\left.K\right)$. The same approach of [28] shows that if $\left(a_{n}\right) \subset \mathbb{C}^{*}$ is an unbounded sequence then $\left(C_{z \mapsto a_{n} z}\right)$ is $\mathcal{M}$-universal on $H\left(\mathbb{C}^{*}\right)$, that is, there is an $f \in H\left(\mathbb{C}^{*}\right)$ whose sequence of dilation-rotations $\left\{f\left(a_{n} \cdot\right)\right\}_{n \geq 1}$ is $\mathcal{M}$-dense in $H\left(\mathbb{C}^{*}\right)$.

Denote by $\operatorname{Aut}(G)$ the group of holomorphic automorphisms of a domain $G$. Recall that $\operatorname{Aut}(\mathbb{C})=\left\{z \mapsto a z+b: a \in \mathbb{C}^{*}, b \in \mathbb{C}\right\}$ and $\operatorname{Aut}\left(\mathbb{C}^{*}\right)=\{z \mapsto a z: a \in$ $\left.\mathbb{C}^{*}\right\} \cup\left\{z \mapsto a / z: a \in \mathbb{C}^{*}\right\}$. Birkhoff's and Zappa's results have been extended or improved in several directions. Restricting ourselves to $\mathbb{C}$ and $\mathbb{C}^{*}$, we remark the following:

(a) In 1995, Montes and the author [7] proved that, for a sequence $\left\{z \stackrel{\varphi_{n}}{\mapsto} a_{n} z+b_{n}\right\}_{n \geq 1} \subset \operatorname{Aut}(\mathbb{C})$, the sequence of composition operators $C_{\varphi_{n}}$ : $f \mapsto f\left(a_{n} \cdot+b_{n}\right)(n \geq 1)$ is universal on $H(\mathbb{C})$ if and only if the sequence

$$
\left\{\min \left\{\left|b_{n}\right|,\left|b_{n} / a_{n}\right|\right\}\right\}_{n \geq 1}
$$

is unbounded. If this is the case, then there is a residual set of universal entire functions. In particular, the composition operator $f \mapsto f(a \cdot+b)$ associated to the automorphism $z \mapsto a z+b$ is hypercyclic if and only if $a=1$ and $b \neq 0$. Moreover, given $\left(a_{n}\right) \subset \mathbb{C}^{*}$, the sequence of composition operators $f \in H\left(\mathbb{C}^{*}\right) \mapsto f\left(a_{n} \cdot\right) \in H\left(\mathbb{C}^{*}\right)$ is $\mathcal{M}$-universal if and only if the sequence

$$
\left\{\max \left\{\left|a_{n}\right|,\left|1 / a_{n}\right|\right\}_{n \geq 1}\right.
$$

is unbounded, in which case there is a residual subset in $H\left(\mathbb{C}^{*}\right)$ of $\mathcal{M}$-universal functions. In particular, the operator $f \mapsto f(a \cdot)$ is $\mathcal{M}$-hypercyclic on $H\left(\mathbb{C}^{*}\right)$ if and only if $|a| \neq 1$. By contrast, no sequence $\left(\varphi_{n}\right) \subset \operatorname{Aut}\left(\mathbb{C}^{*}\right)$ generates a universal sequence $\left(C_{\varphi_{n}}\right)$ on $H\left(\mathbb{C}^{*}\right)$, see [7] and [19].

(b) In 1996, Luh [23] improved Zappa's theorem by constructing, for a given unbounded sequence $\left(a_{n}\right) \subset \mathbb{C}^{*}$, an entire function $f$ such that $\left\{f\left(a_{n} \cdot\right)\right\}_{n \geq 1}$ is $\mathcal{M}$-dense in $H\left(\mathbb{C}^{*}\right)$. The set of such functions $f$ is in fact residual in $H(\mathbb{C})$ [8]. Notice that the unboundedness of $\left(a_{n}\right)$ is necessary for the existence of these multiplicatively universal entire functions, for if $\left|a_{n}\right| \leq M<+\infty$ $(n \geq 1)$ then the sequence $\left(f\left(a_{n} \cdot\right)\right)$ cannot approximate the constant function $1+\sup _{|z| \leq M}|f(z)|$ on the compact set $\{1\}$.

In view of (a)-(b), a natural question arises: which sequences $\left\{a_{n} z+b_{n}\right\}_{n \geq 1} \subset$ $\operatorname{Aut}(\mathbb{C})$ support entire functions $f$ satisfying that $\left\{f\left(a_{n} \cdot+b_{n}\right)\right\}_{n \geq 1}$ is $\mathcal{M}$-dense in $H\left(\mathbb{C}^{*}\right)$ ? We provide necessary as well as sufficient conditions in Section 2.

In the last few years, a number of authors have invested much effort in making universality compatible with some additional property, specially boundedness on large sets. In this respect, Tenthoff [26] proved in 2000 that there are Birkhoffuniversal entire functions that are bounded on every (straight) line. M.C. Calderón [13] (see also [17]) showed in 2002 the existence of a dense linear manifold of Birkhoff-universal entire functions $f$ (see [10] for similar results in the harmonic setting) which are bounded on any sector $\{z: 0 \leq \arg z \leq \alpha\} \quad(0<\alpha<2 \pi)$ and any strip (i.e. any domain lying between two parallel lines); even $\exp \left(|z|^{\alpha}\right) f(z) \rightarrow 0$ 
$(z \rightarrow \infty)$ on these subsets for fixed $\alpha \in(0,1 / 2)$, and many more additional properties hold. In 2003, Costakis and Sambarino [14] proved that there are $\tau_{1^{-}}$ hypercyclic entire functions $f$ tending to 0 as $z \rightarrow \infty$ on each sector $\{z: \varepsilon \leq$ $\arg z \leq 2 \pi-\varepsilon\}(0<\varepsilon<\pi)$. In 2006, Niess [25] gave the following necessary and sufficient condition for the existence of $\left(\tau_{a_{n}}\right)$-universal entire functions bounded on every line: there exists a subsequence $\left(a_{n_{k}}\right)$ such that, for every $R>0$ and every line $L$, there is $k_{0} \in \mathbb{N}$ with $L \cap B\left(a_{n_{k}}, R\right)=\emptyset$ for all $k \geq k_{0}$ (as usual, $B(a, R):=\{z \in \mathbb{C}:|z-a|<r\}$ and $\bar{B}(a, R):=\{z \in \mathbb{C}:|z-a|<r\})$. Gharibyan, Luh and Niess [17, Theorem 1.2] established the existence of an entire function $\varphi$ and of sequences $\left(a_{n}\right),\left(b_{n}\right) \subset \mathbb{C}$ with $a_{n} \rightarrow 0, b_{n} \rightarrow \infty$ such that $\varphi$ is $\left(C_{a_{n} z+b_{n}}\right)$-universal and bounded on every line. Bonilla and the author [5] proved that, for given $F \subset \mathbb{C}$, there exist a Birkhoff-universal entire function that is bounded on $F$ if and only if there exists an Arakelian subset $F_{0}$ of $\mathbb{C}$ (i.e. $F_{0}$ is closed and $\mathbb{C}_{\infty} \backslash F_{0}$ is connected and locally connected at $\infty$, where $\mathbb{C}_{\infty}$ is the onepoint compactification of $\mathbb{C})$ such that $\rho_{i}\left(\mathbb{C} \backslash F_{0}\right)=+\infty\left(\right.$ here $\rho_{i}(A)=\sup \{r>0$ : there exists a closed ball $B$ of radius $r$ with $B \subset A\}$, the inscribed radius of a subset $A \subset \mathbb{C}$ ). In 2010, Calderón, Luh and the author [6] stated the following: if $A \subset \mathbb{C}$ is an unbounded Arakelian set with $\rho_{i}(\mathbb{C} \backslash A)=+\infty$, there is a dense linear manifold $M$ of entire functions all of whose nonzero members are Birkhoffuniversal and $\exp \left(|z|^{\alpha}\right) f(z) \rightarrow 0(z \rightarrow \infty, z \in A)$ for all $\alpha<1 / 2$ and $f \in M$. Passing to $\mathbb{C}^{*}, \mathrm{~A}$. Vogt [27] has recently constructed a multiplicative universal entire function $\varphi$ with respect to a given unbounded sequence $\left(a_{n}\right)$ such that $\varphi$ is bounded on some curve $\Gamma$ tending to $\infty$. On the contrary, he has proved that any such $\varphi$ is necessarily unbounded on every line.

In Section 3, we investigate boundedness and unboundedness on large subsets of $\mathbb{C}$ for entire functions being compositionally universal $(\mathcal{M}$-universal) on $H(\mathbb{C})$ (on $H\left(\mathbb{C}^{*}\right)$, resp.) with respect to sequences in $\operatorname{Aut}(\mathbb{C})$.

In 2006, Bayart and Grivaux [3] presented the more stringent notion of frequent hypercyclicity, that is a kind of quantified hypercyclicity. If $X$ is a topological vector space, then an operator $T \in L(X)$ is said to be frequent hypercyclic provided that there is a vector $x \in X$, called frequent hypercyclic for $T$, such that, for every nonempty open set $U \subset X$, the set $\left\{n \in \mathbb{N}: T^{n} x \in U\right\}$ has lower positive density, that is,

$$
\liminf _{n \rightarrow \infty} \frac{\operatorname{card}\left\{k \in\{1, \ldots, n\}: T^{k} x \in U\right\}}{n}>0 .
$$

As in the mere hypercyclicity, the concept can be extended to a sequence of continuous mappings $T_{n}: X \rightarrow Y(n \in \mathbb{N})$ between two topological spaces $X, Y$ (simply replace $T^{k} x$ by $T_{k} x$ in (2)), in which case $\left(T_{n}\right)$ is called frequent universal, and $x$ a frequent universal element for $\left(T_{n}\right)$, see [12]. Sufficient conditions for frequent hypercyclicity/universality have been given in [3] and [12]. In [3] it is proved the frequent universality of any translation operator $\tau_{a}(a \neq 0)$ on $H(\mathbb{C})$. This is also shown by Bonilla and Grosse-Erdmann in three different ways (see [11], [12, Example 3.2] and [12, Theorem 4.2]) as a consequence of more general criteria. 
Our third and final goal, which will be performed in Section 4, is to extend this result by analyzing the frequent universality of a sequence of composition operators on $H(\mathbb{C})$ generated by a sequence $\left(\varphi_{n}\right) \subset \operatorname{Aut}(\mathbb{C})$.

\section{2. $\mathcal{M}$-universal entire functions}

Our first theorem extends and unifies the assertions given by Luh, Prado and Bernal about existence of entire functions that are $\mathcal{M}$-universal on $H\left(\mathbb{C}^{*}\right)$ with respect to sequences of composition operators generated by automorphisms of $\mathbb{C}$, see Section 1. As usual, we adopt the convention $\alpha / 0=+\infty$ whenever $\alpha \in(0,+\infty)$.

Theorem 2.1. Assume that $\left\{\varphi_{n}\right\}_{n \geq 1} \subset \operatorname{Aut}(\mathbb{C})$ and $\varphi_{n}(z)=a_{n} z+b_{n}(n \geq 1)$.

(a) If the sequence

$$
\left\{\max \left\{\min \left\{\left|a_{n}\right|,\left|a_{n} / b_{n}\right|\right\}, \min \left\{\left|b_{n}\right|,\left|b_{n} / a_{n}\right|\right\}\right\}\right\}_{n \geq 1}
$$

is unbounded then the sequence $C_{\varphi_{n}}: H(\mathbb{C}) \rightarrow H\left(\mathbb{C}^{*}\right)(n \in \mathbb{N})$ is $\mathcal{M}$ universal. In fact, the set $\mathcal{U}_{\mathcal{M}}\left(\left(C_{\varphi_{n}}\right)\right):=\left\{f \in H(\mathbb{C}):\left(f \circ \varphi_{n}\right)\right.$ is $\mathcal{M}$-dense in $\left.H\left(\mathbb{C}^{*}\right)\right\}$ is a residual subset of $H(\mathbb{C})$.

(b) If the sequence $C_{\varphi_{n}}: H(\mathbb{C}) \rightarrow H\left(\mathbb{C}^{*}\right)(n \in \mathbb{N})$ is $\mathcal{M}$-universal then

(i) at least one of the sequences $\left(a_{n}\right),\left(b_{n}\right)$ is unbounded, and

(ii) there exists a sequence $\left\{n_{1}<n_{2}<\cdots\right\} \subset \mathbb{N}$ such that the sequence of zeros $\left\{\varphi_{n_{k}}^{-1}(0)\right\}_{k \geq 1}$ is not contained in any set $K \in \mathcal{M}\left(\mathbb{C}^{*}\right)$.

Proof. (a) Assume that (3) is unbounded. Then at least one of the sequences $\left\{\min \left\{\left|a_{n}\right|,\left|a_{n} / b_{n}\right|\right\}\right\}_{n \geq 1},\left\{\min \left\{\left|b_{n}\right|,\left|b_{n} / a_{n}\right|\right\}\right\}_{n \geq 1}$ is unbounded. If the latter one is unbounded, then by [7, Proposition 2.3 and Theorem 3.1] the set $\mathcal{U}\left(\left(C_{\varphi_{n}}\right)\right)=\{f \in$ $H(\mathbb{C})$ : the set $\left\{f \circ \varphi_{n}\right\}_{n \geq 1}$ is dense in $\left.H(\mathbb{C})\right\}$ is a residual subset of $H(\mathbb{C})$. But by Runge's theorem $H(\mathbb{C})$ is $\mathcal{M}$-dense in $H\left(\mathbb{C}^{*}\right)$. Therefore $\mathcal{U}_{\mathcal{M}}\left(\left(C_{\varphi_{n}}\right)\right) \supset \mathcal{U}\left(\left(C_{\varphi_{n}}\right)\right)$, so $\mathcal{U}_{\mathcal{M}}\left(\left(C_{\varphi_{n}}\right)\right)$ is residual in $H(\mathbb{C})$. Suppose now that $\left\{\min \left\{\left|a_{n}\right|,\left|a_{n} / b_{n}\right|\right\}\right\}_{n \geq 1}$ is unbounded. Fix a compact set $K \subset \mathbb{C}$, a number $\varepsilon>0$, a set $L \in \mathcal{M}\left(\mathbb{C}^{*}\right)$ and functions $f \in H(\mathbb{C}), g \in H\left(\mathbb{C}^{*}\right)$. Consider the sets $U(f, \varepsilon, K):=\{h \in H(\mathbb{C})$ : $|h(z)-f(z)|<\varepsilon$ for all $z \in K\}$ and $V(g, \varepsilon, L):=\left\{h \in H\left(\mathbb{C}^{*}\right):|h(z)-g(z)|<\varepsilon\right.$ for all $z \in L\}$, which are respectively basic open sets in $H(\mathbb{C})$ and $H\left(\mathbb{C}^{*}\right)$. Choose $r, s>0$ with $K \subset \bar{B}(0, r)$ and $L \cap \bar{B}(0,2 s)=\emptyset$, then select $n \in \mathbb{N}$ such that $\left|a_{n}\right|>r / s$ and $\left|a_{n} / b_{n}\right|>1 / s$. Then we obtain, for all $z \in L$,

$$
\left|\varphi_{n}(z)\right|=\left|a_{n} z+b_{n}\right|=\left|a_{n}\right|\left|z+\frac{b_{n}}{a_{n}}\right|>\frac{r}{s}(2 s-s)=r .
$$

Hence $\bar{B}(0, r) \cap \varphi_{n}(L)=\emptyset$. Define the function $F: \bar{B}(0, r) \cup \varphi_{n}(L) \rightarrow \mathbb{C}$ by

$$
F(z)=\left\{\begin{array}{cl}
f(z) & \text { if } z \in \bar{B}(0, r) \\
g\left(\frac{z-b_{n}}{a_{n}}\right) & \text { if } z \in \varphi_{n}(L)
\end{array}\right.
$$

Note that $S:=\bar{B}(0, r) \cup \varphi_{n}(L) \in \mathcal{M}(\mathbb{C})$ because $\varphi_{n} \in \operatorname{Aut}(\mathbb{C})$. Moreover, $F$ is holomorphic on a neighborhood of $S$. By Runge's theorem, there exists a polynomial $h$ (so $h \in H(\mathbb{C})$ ) with $|h(z)-F(z)|<\varepsilon$ for all $z \in S$. In particular, $|h(z)-f(z)|<\varepsilon$ 
for all $z \in K$ and $\left|h\left(\varphi_{n}(z)\right)-g(z)\right|<\varepsilon$ for all $z \in L$. In other words, $h \in U(f, \varepsilon, K)$ and $C_{\varphi_{n}} h \in V(g, \varepsilon, L)$. Therefore $C_{\varphi_{n}}(U(f, \varepsilon, K)) \cap V(g, \varepsilon, L) \neq \emptyset$, whence each set $\bigcup_{n \in \mathbb{N}} C_{\varphi_{n}}^{-1}(V(g, \varepsilon, L))$ is (open and) dense in $H(\mathbb{C})$. Now, for every domain $G$ there exists a sequence $\left\{K_{j}\right\}_{j \geq 1} \subset \mathcal{M}(G)$ that is exhaustive, that is, given $L \in \mathcal{M}(G)$ there is $j_{0} \in \mathbb{N}$ with $L \subset K_{j_{0}}$ [7, Lemma 2.9]. For the special case $G=\mathbb{C}^{*}$, it follows that

$$
\mathcal{U}_{\mathcal{M}}\left(\left(C_{\varphi_{n}}\right)\right)=\bigcap_{\substack{g \in H\left(\mathbb{C}^{*}\right) \\ L \in \mathcal{M}, \varepsilon>0}} \bigcup_{n \in \mathbb{N}} C_{\varphi_{n}}^{-1}(V(g, \varepsilon, L))=\bigcap_{k, l, j \in \mathbb{N}} \bigcup_{n \in \mathbb{N}} C_{\varphi_{n}}^{-1}\left(V\left(g_{k}, 1 / l, K_{j}\right)\right),
$$

where $\left(g_{k}\right)$ is any fixed dense sequence in $H\left(\mathbb{C}^{*}\right)$. Since $H(\mathbb{C})$ is a Baire space, we obtain that $\mathcal{U}_{\mathcal{M}}\left(\left(C_{\varphi_{n}}\right)\right)$ is a dense $G_{\delta}$ (so residual) subset of $H(\mathbb{C})$.

(b) Suppose that there exists $f \in H(\mathbb{C})$ that is $\mathcal{M}$-universal for the sequence $C_{\varphi_{n}}: H(\mathbb{C}) \rightarrow H\left(\mathbb{C}^{*}\right)(n \in \mathbb{N})$. By way of contradiction, assume also that $\left(a_{n}\right)$ and $\left(b_{n}\right)$ are bounded. Then $\left|a_{n}\right|+\left|b_{n}\right| \leq M<+\infty(n \in \mathbb{N})$, say. Therefore no sequence $\left(f\left(a_{n_{k}} z+b_{n_{k}}\right)\right)$ would be able to approximate the constant function $1+\max _{|w| \leq M}|f(w)|$ on the compact set $\{1\} \in \mathcal{M}\left(\mathbb{C}^{*}\right)$, so contradicting the hypothesis. Thus, (i) is satisfied. Suppose now, again by way of contradiction, that (ii) does not hold. By hypothesis, there is $f \in H(\mathbb{C})$ as well as a sequence $\left\{n_{1}<n_{2}<\cdots\right\} \subset \mathbb{N}$ such that $f\left(a_{n_{k}} z+b_{n_{k}}\right) \rightarrow g(z)(k \rightarrow \infty)$ uniformly on each member of $\mathcal{M}\left(\mathbb{C}^{*}\right)$, where $g(z):=1+|f(0)|$. But one can find a set $K \in \mathcal{M}\left(\mathbb{C}^{*}\right)$ with $\left\{\varphi_{n_{k}}^{-1}(0)=-b_{n_{k}} / a_{n_{k}}\right\}_{n \geq 1} \subset K$. Then

$$
\sup _{z \in K}\left|f\left(a_{n_{k}} z+b_{n_{k}}\right)-g(z)\right| \geq\left|f\left(\varphi_{n_{k}}\left(\varphi_{n_{k}}^{-1}(0)\right)\right)-g(z)\right|=|f(0)-(1+|f(0)|)| \geq 1
$$

for all $k \in \mathbb{N}$, which is absurd. This proves the theorem.

Corollary 2.2. Let $\varphi \in \operatorname{Aut}(\mathbb{C})$, with $\varphi(z)=a z+b$. Then the following properties are equivalent:

(a) There is an entire function $f$ whose orbit $\left\{f \circ \varphi^{[n]}\right\}_{n \geq 1}$ is $\mathcal{M}$-dense in $H\left(\mathbb{C}^{*}\right)$.

(b) The set $\mathcal{U}_{\mathcal{M}}\left(\left(C_{\left.\varphi^{[n]}\right]}\right)\right)$ is residual in $H(\mathbb{C})$.

(c) Either $|a|>1$ and $b=0$ or $a=1$ and $b \neq 0$.

Proof. It is evident that (b) implies (a). Suppose that (c) holds. If $a=1$ and $b \neq 0$ (i.e. $\varphi$ is a translation) then we have in fact that $C_{\varphi}$ is hypercyclic on $H(\mathbb{C})$, so the sequence $\left(C_{\varphi}^{n}\right)=\left(C_{\varphi^{[n]}}\right)$ is transitive from $H(\mathbb{C})$ into itself. Therefore the set $\mathcal{U}\left(\left(C_{\varphi}^{n}\right)\right)$ of its hypercyclic functions is residual in $H(\mathbb{C})$. But, again by Runge's theorem, $\mathcal{U}\left(\left(C_{\varphi}^{n}\right)\right) \subset \mathcal{U}_{\mathcal{M}}\left(\left(C_{\varphi^{[n]}}\right)\right)$, from which the residuality of $\mathcal{U}_{\mathcal{M}}\left(\left(C_{\varphi^{[n]}}\right)\right)$ follows. If $a \neq 1$ then $\varphi^{[n]}(z)=a^{n} z+a^{n-1} b+a^{n-2} b+\cdots+a b=a_{n} z+b_{n}$, where $a_{n}=a^{n}$ and $b_{n}=b \cdot \frac{a^{n}-1}{a-1}$. Therefore, if $|a|>1$ and $b=0$, the sequence $\left\{\min \left\{\left|a_{n}\right|,\left|a_{n} / b_{n}\right|\right\}_{n>1}=\left\{|a|^{n}\right\}_{n>1}\right.$ is unbounded. By Theorem 2.1, (b) is satisfied. Finally, if (a) is true and $a \neq 1$ then by Theorem 2.1 at least one of the sequences $\left(a^{n}\right),\left(b \frac{a^{n}-1}{a-1}\right)$ is unbounded, hence $|a|>1$. If $a \neq 1$ and $b \neq 0$ then $\left(\varphi^{[n]}\right)^{-1}(0)=\frac{b\left(1-a^{-n}\right)}{1-a} \underset{n}{\longrightarrow} \frac{b}{1-a} \in \mathbb{C}^{*}$, so the full sequence $\left\{\left(\varphi^{[n]}\right)^{-1}(0)\right\}_{n \geq 1}$ is contained in the set $K:=\left\{\frac{b}{1-a}\right\} \cup\left\{\left(\varphi^{[n]}\right)^{-1}(0): n \in \mathbb{N}\right\} \in \mathcal{M}\left(\mathbb{C}^{*}\right)$. According to 
Theorem 2.1, there is no entire function $f$ such that $\left\{f \circ \varphi^{[n]}\right\}_{n \geq 1}$ is $\mathcal{M}$-dense. If $a=1$ and $b=0$ then $\varphi$ is the identity, whose iterates clearly do not generate compositional universality. Hence (a) implies (c).

Remark 2.3. 1. As an example, there is no entire function $f$ such that the set $A(f):=\left\{z \mapsto f\left(n^{2} z+n\right): n \in \mathbb{N}\right\}$ is dense in $H(\mathbb{C})$, because the sequence (1) (see Section 1) for $a_{n}=n^{2}, b_{n}=n$ is $\{1 / n\}_{n \in \mathbb{N}}$, which is bounded. Nevertheless, entire functions $f$ such that $A(f)$ is $\mathcal{M}$-dense in $H\left(\mathbb{C}^{*}\right)$ do exist: indeed, $\min \left\{\left|a_{n}\right|,\left|a_{n} / b_{n}\right|\right\}=n(n \in \mathbb{N})$ and Theorem 2.1 applies.

2. Let $\left(q_{n}\right)$ be an enumeration of the set $\left\{e^{i t}: t \in \mathbb{Q}\right\}(\mathbb{Q}=$ the set of rational numbers), which is dense in the unit circle $\mathbb{T}$. Then $\varphi_{n}(z)=a_{n} z+b_{n}:=$ $n z+n q_{n} \in \operatorname{Aut}(\mathbb{C})(n \geq 1)$ satisfies that the sequences (1) and (3) are bounded, $\left(a_{n}\right)$ and $\left(b_{n}\right)$ are unbounded and the full sequence $\left(\varphi_{n}^{-1}(0)\right)=\left(-q_{n}\right)$ is not contained in any member of $\mathcal{M}\left(\mathbb{C}^{*}\right)$. Hence $\left(C_{\varphi_{n}}\right)$ is not $H(\mathbb{C})$-universal but we can extract from Theorem 2.1 neither the $\mathcal{M}$-universality nor the non- $\mathcal{M}$-universality of $\left(C_{\varphi_{n}}\right)$ on $H\left(\mathbb{C}^{*}\right)$. This raises the question of finding an intermediate property between the unboundedness of (3) and (i) +(ii) of Theorem 2.1 characterizing the $\mathcal{M}$-universality of $\left\{H(\mathbb{C}) \stackrel{C_{\varphi_{n}}}{\longrightarrow} H\left(\mathbb{C}^{*}\right)\right\}_{n \geq 1}$.

\section{Bounded universal entire functions}

In this section we show that, under appropriate conditions, no $\mathcal{M}$-universal entire function on $H\left(\mathbb{C}^{*}\right)$ is bounded on every member of a large family of unbounded sets (including lines), see Theorem 3.1. In the positive direction, it will be proved (Theorem 3.3) the existence of an $\mathcal{M}$-universal entire function $f$ for which there is an unbounded set $\Gamma$ such that $f$ tends to 0 as $z \rightarrow \infty$ (in particular, $f$ is bounded) on it. We may even prescribe the set $\Gamma$ within a large family, including again all lines, in the case of $H(\mathbb{C})$-universality, see Theorem 3.5. This improves recent results by A. Vogt [27] and completes a number of findings by several authors, see Section 1.

We introduce the following notion. By $\mathcal{S}_{0}$ we denote the collection of all closed sectors $S$ with vertex at the origin and amplitude $\operatorname{ampl}(S) \in(0,2 \pi)$. We say that a set $\Gamma$ is totally non-spiral-like provided that there are two unbounded connected sets $\Gamma_{0}, L$ and a sector $S \in \mathcal{S}_{0}$ such that $\Gamma_{0} \subset \Gamma, L \subset S$ and $\Gamma_{0} \cap L=\emptyset$. For instance, every set $\Gamma$ with non-total oscillation near $\infty$ (i.e. such that there are $R>0, S \in \mathcal{S}_{0}$ and an unbounded connected set $\Gamma_{0} \subset \Gamma$ with $\left.\Gamma_{0} \cap S \cap\{|z|>R\}=\emptyset\right)$ is totally non-spiral-like. In particular, every line, every parabola, and in general every unbounded algebraic curve $P(x, y)=0(P=$ a nonconstant polynomial of two real variables with real coefficients) are totally non-spiral-like sets.

Theorem 3.1. Assume that $\left\{\varphi_{n}(z)=a_{n} z+b_{n}\right\}_{n \geq 1} \subset \operatorname{Aut}(\mathbb{C})$ is a sequence such that $a_{n} \rightarrow \infty$ and $b_{n} / a_{n} \rightarrow 0$ as $n \rightarrow \infty$. Let $\Gamma$ be a totally non-spiral-like subset of $\mathbb{C}$. Then every $\mathcal{M}$-universal entire function for the sequence $C_{\varphi_{n}}: H(\mathbb{C}) \rightarrow H\left(\mathbb{C}^{*}\right)$ $(n \geq 1)$ is unbounded on $\Gamma$. 
Proof. Consider the compact set given by the "snail" curve

$$
K:=\left\{z(\theta)=\left(1+\frac{\theta}{2 \pi}\right) e^{i \theta} \in \mathbb{C}: \theta \in[0,6 \pi]\right\} .
$$

Observe that $K \in \mathcal{M}\left(\mathbb{C}^{*}\right)$ and note that any ray $\sigma$ starting from any point in $\mathbb{D}$ intersects $K$ at least at two different points $z\left(\theta_{1, \sigma}\right), z\left(\theta_{2, \sigma}\right)$ with $\theta_{2, \sigma}-\theta_{1, \sigma}>\pi$ and $\left|z\left(\theta_{1, \sigma}\right)\right|>1,\left|z\left(\theta_{2, \sigma}\right)\right|>2$. It follows that if $M$ is an unbounded, connected set lying in a non-degenerated closed sector $\Sigma$ whose vertex is at $\mathbb{D}$ with $M \cap \mathbb{D} \neq$ $\emptyset$, then $M$ must intersect both curvilinear arcs of the closed annular "sector" determined by $K$ and the segments joining the pair of points $\left(z\left(\theta_{1, \sigma}\right), z\left(\theta_{2, \sigma}\right)\right)$ and $\left(z\left(\theta_{1, \lambda}\right), z\left(\theta_{2, \lambda}\right)\right)$, where $\sigma$ and $\lambda$ are the rays forming the boundary of $\Sigma$ (there may be more than one of such annular sectors; then simply select one of them). As a consequence, if $A$ is an unbounded connected subset of $\mathbb{C}$ such that $A \cap \mathbb{D} \neq \emptyset=A \cap M$, then $A$ should intersect the snail, i.e. $A \cap K \neq \emptyset$. This property will be used later.

Notice that by Theorem 2.1, there are in fact $\mathcal{M}$-universal entire functions for $\left(C_{\varphi_{n}}\right)$. Let $f$ be one of them and assume, by way of contradiction, that $f$ is bounded on $\Gamma$, say $|f(z)| \leq \alpha(z \in \Gamma)$. By universality, there is a sequence $\left\{n_{1}<n_{2}<\cdots\right\} \subset \mathbb{N}$ such that $C_{\varphi_{n_{k}}} f$ tends to the constant function $1+\alpha$ uniformly on $K$ as $k \rightarrow \infty$. Without loss of generality, we may assume that $\left(n_{k}\right)$ is the full sequence $\mathbb{N}$. In particular, there is $n_{0} \in \mathbb{N}$ such that

$$
\sup _{z \in K}\left|f\left(a_{n} z+b_{n}\right)-(1+\alpha)\right|<1 \text { for all } n \geq n_{0} .
$$

Let $\Gamma_{0}, L, S$ the sets supplied by the definition of non-total spiral-likeness for our set $\Gamma$. Fix any $z_{0} \in \Gamma_{0}$ and any $w_{0} \in L$. Since $a_{n} \rightarrow \infty$ and $b_{n} / a_{n} \rightarrow 0$, there is $m \in \mathbb{N}$ with $m \geq n_{0}$ such that $\left|z_{0} / a_{m}\right|<1 / 2,\left|w_{0} / a_{m}\right|<1 / 2$ and $\left|b_{m} / a_{m}\right|<1 / 2$. Then $\frac{z_{0}-b_{m}}{a_{m}}, \frac{w_{0}-b_{m}}{a_{m}} \in \mathbb{D}$. We have:

- The sets $A:=\left\{\frac{z-b_{m}}{a_{m}}: z \in \Gamma_{0}\right\}$ and $M:=\left\{\frac{z-b_{m}}{a_{m}}: z \in L\right\}$ are unbounded connected sets, and $\Sigma:=\left\{\frac{z-b_{m}}{a_{m}}: z \in S\right\}$ is a closed sector with $\operatorname{ampl}(\Sigma)$ $\in(0,2 \pi)$ and vertex at the point $-b_{m} / a_{m} \in \mathbb{D}$.

- $A \cap \mathbb{D} \neq \emptyset \neq M \cap \mathbb{D}, A \cap M=\emptyset$ and $M \subset \Sigma$.

From the first paragraph, $A \cap K \neq \emptyset$. Therefore there exist $z_{1} \in K$ and $z_{2} \in \Gamma_{0}$ (so $z_{2} \in \Gamma$ ) such that $z_{1}=\frac{z_{2}-b_{m}}{a_{m}}$. It follows from (4) that $\left|f\left(a_{m} z_{1}+b_{m}\right)-(1+\alpha)\right|<$ 1. Hence $\left|f\left(z_{2}\right)-(1+\alpha)\right|<1$, so $\left|f\left(z_{2}\right)\right|>\alpha$ due to the triangular inequality. This contradicts the fact that $|f| \leq \alpha$ on $\Gamma$.

Remark 3.2. If the snail $K$ of the last proof had only two turns (i.e. $\theta \in[0,4 \pi]$ ) then not every ray $\sigma$ with vertex at a point of $\mathbb{D}$ would intersect at least twice the set $K$ : take for instance $\sigma=\{z=t+i(-1+(t / 2)): t \geq 0\}$.

In the next result, notice that similarly to Theorem 3.1 the condition imposed on $\left(a_{n}\right),\left(b_{n}\right)$ guarantees $\mathcal{M}$-universality on $H\left(\mathbb{C}^{*}\right)$, but discards $H(\mathbb{C})$-universality. By contrast, the restriction imposed in Theorem 3.4 entails $H(\mathbb{C})$-universality for the sequence $\left(C_{\varphi_{n}}\right)$, with $\varphi_{n}(z)=a_{n} z+b_{n}$. 
Theorem 3.3. Let $\left\{\varphi_{n}(z)=a_{n} z+b_{n}\right\}_{n \geq 1} \subset \operatorname{Aut}(\mathbb{C})$. Suppose that the sequence $\left\{\min \left\{\left|a_{n}\right|,\left|a_{n} / b_{n}\right|\right\}\right\}_{n \geq 1}$ is unbounded. Let $\omega: \mathbb{C} \rightarrow(0,+\infty)$ be a continuous function. Then there exists an $\mathcal{M}$-universal entire function $f$ with respect to $\left\{C_{\varphi_{n}}\right.$ : $\left.H(\mathbb{C}) \rightarrow H\left(\mathbb{C}^{*}\right)\right\}_{n \geq 1}$ that admits an unbounded connected set $\Gamma \subset \mathbb{C}$ such that $\lim _{\substack{z \rightarrow \infty \\ z \in \Gamma}} \omega(z) f(z)=0$.

Proof. We will follow the approach of [27] in the first steps. Choose an exhaustive sequence $\left\{K_{n}\right\}_{n>1}$ in $\mathcal{M}\left(\mathbb{C}^{*}\right)$ as in the proof of Theorem 2.1 (see [7, Lemma 2.9] or $[23$, Lemma 3$]$ ). Let $\left\{Q_{n}\right\}_{n \geq 1}$ be an enumeration of all polynomials whose coefficients are in $\mathbb{Q}+i \mathbb{Q}$. In addition, let $\left\{\left(Q_{n}^{*}, K_{n}^{*}\right)\right\}_{n>1}$ be a sequence with the property that every pair $\left(Q_{i}, K_{j}\right)(i, j \in \mathbb{N})$ occurs infinitely many often.

By hypothesis, and without loss of generality, one can assume that $\min \left\{\left|a_{n}\right|\right.$, $\left.\left|a_{n} / b_{n}\right|\right\} \rightarrow+\infty$ as $n \rightarrow \infty$. Then $a_{n} \rightarrow \infty$ and $b_{n} / a_{n} \rightarrow 0$. Therefore, given $r, s \in(0,+\infty)$, one has

$$
\left|a_{n}\right|\left(r-\left|b_{n} / a_{n}\right|\right)>s \text { for } n \text { large enough. }
$$

Set $n_{1}=1$. Proceeding by induction, and assuming that $n_{1}<n_{2}<\cdots<n_{k}$ have been already determined, we can choose due to (5) an integer $n_{k+1}>n_{k}$ such that

$$
\left|a_{n_{k+1}}\right|\left(\inf \left\{|z|: z \in K_{k}^{*}\right\}-\left|b_{n_{k+1}} / a_{n_{k+1}}\right|\right)>1+\sup \left\{|z|: z \in a_{n_{k}} K_{k}^{*}+b_{n_{k}}\right\} .
$$

With the help of the triangular inequality, (6) shows that the sets $A_{k}:=a_{n_{k}} K_{k}^{*}+$ $b_{n_{k}}(k \in \mathbb{N})$ are mutually disjoint. Of course, each $A_{k}$ is compact and has connected complement. In addition, due to (6), one gets $\operatorname{dist}\left(A_{j}, A_{k}\right) \geq 1(j, k \in \mathbb{N} ; j \neq k)$. Then $\bigcup_{k \in \mathbb{N}} A_{k}$ is closed and $G:=\mathbb{C} \backslash \bigcup_{k \in \mathbb{N}} A_{k}$ is an unbounded connected open subset of $\mathbb{C}$. Hence we can find an unbounded regular curve $\Gamma$ in $G$, that is, $\Gamma$ is the image of some differentiable function $\gamma:[0,+\infty) \rightarrow G$ with $\lim _{t \rightarrow \infty} \gamma(t)=\infty$. In particular, $\Gamma$ is an unbounded connected closed subset of $\mathbb{C}$ with empty interior $\Gamma^{0}$.

Consider the set $A:=G \cup \bigcup_{k \in \mathbb{N}} A_{k}$. Then $A$ is a Carleman set, that is, it satisfies the following properties:

- $A$ is closed.

- $\mathbb{C}_{\infty} \backslash A$ is connected and locally connected at $\infty$.

- For every compact set $K \subset \mathbb{C}$ there is a neighborhood $V$ of $\infty$ in $\mathbb{C}_{\infty}$ such that no component of $A^{0}$ intersects both $K$ and $V$.

Define the function $g: A \rightarrow \mathbb{C}$ by

$$
g(z)=\left\{\begin{array}{cl}
0 & \text { if } z \in \Gamma \\
Q_{k}^{*}\left(\frac{z-b_{n_{k}}}{a_{n_{k}}}\right) & \text { if } z \in A_{k}(k \in \mathbb{N}) .
\end{array}\right.
$$

This function is evidently holomorphic in $A^{0}$ and continuous on $A$. Moreover, the function $\varepsilon(z)=\left\{\begin{array}{cl}\frac{1}{(1+|z|) \omega(z)} & \text { if } z \in \Gamma \\ \frac{1}{k} & \text { if } z \in A_{k}(k \in \mathbb{N})\end{array} \quad\right.$ is continuous on $A$ and positive. 
According to the Nersesjan tangential approximation theorem (see [16, pp. 155159] or [24]), there exists an entire function $f$ satisfying

$$
|f(z)-g(z)|<\varepsilon(z) \quad(z \in A) .
$$

In particular, $|f(z) \omega(z)|<\frac{1}{1+|z|}$ for all $z \in \Gamma$. Hence $f(z) \omega(z) \rightarrow 0(z \rightarrow \infty ; z \in$ $\Gamma)$.

Finally, we show the $\mathcal{M}$-universality of $f$ for $\left(C_{\varphi_{n}}\right)$. From (7) we get $\mid f(z)-$ $Q_{k}^{*}\left(\frac{z-b_{n_{k}}}{a_{n_{k}}}\right) \mid<\frac{1}{k}$ for all $z \in A_{k}$ or, that is the same, $\left|f\left(a_{n_{k}} z+b_{n_{k}}\right)-Q_{k}^{*}(z)\right|<\frac{1}{k}$ for all $z \in K_{k}^{*}$. Fix $h \in H\left(\mathbb{C}^{*}\right), \varepsilon>0$ and $K \in \mathcal{M}\left(\mathbb{C}^{*}\right)$. Select $n_{0} \in \mathbb{N}$ with $K \subset K_{n_{0}}$. By Runge's approximation theorem there exists $m_{0} \in \mathbb{N}$ with $\left|Q_{m_{0}}(z)-h(z)\right|<$ $\varepsilon / 2$ for all $z \in K$. Now choose a sequence $\{k(1)<k(2)<\cdots\} \subset \mathbb{N}$ such that $\left(Q_{k(j)}^{*}, K_{k(j)}^{*}\right)=\left(Q_{m_{0}}, K_{n_{0}}\right)$ for all $j \geq 1$. Pick $j \in \mathbb{N}$ such that $k(j)>2 / \varepsilon$ and let $\nu:=n_{k(j)}$. Since $K \subset K_{n_{0}}=K_{k(j)}^{*}$ we obtain for all $z \in K$ that

$\left|\left(C_{\varphi_{\nu}} f\right)(z)-h(z)\right| \leq\left|f\left(a_{\nu} z+b_{\nu}\right)-Q_{k(j)}^{*}(z)\right|+\left|Q_{m_{0}}(z)-h(z)\right|<\frac{1}{k(j)}+\frac{\varepsilon}{2}<\varepsilon$.

To summarize, $\left\{C_{\varphi_{n}} f\right\}_{n \geq 1}$ is $\mathcal{M}$-dense in $H\left(\mathbb{C}^{*}\right)$, as required.

Remark 3.4. It is also possible to obtain an $\mathcal{M}$-universal entire function $f$ as in the last theorem and an unbounded connected open set $\Gamma \subset \mathbb{C}$ such that $\lim \underset{\substack{z \in \Gamma \\ z \in \Gamma}}{ } \omega(z) f(z)=0$ for prescribed functions $\omega(z)$, but within a more restrictive class of weight functions, see the approach of Theorem 3.5.

The following theorem extends the results on bounded universal functions by Niess [25], Bernal and Bonilla [5] and Gharibyan, Luh and Niess [17, Theorem 1.2] given in Section 1.

Theorem 3.5. Let $\Gamma \subset \mathbb{C}$ be an unbounded subset. Assume that $\left\{\varphi_{n}(z)=a_{n} z+\right.$ $\left.b_{n}\right\}_{n \geq 1} \subset \operatorname{Aut}(\mathbb{C})$ and that $b_{n} \rightarrow \infty$ and $b_{n} / a_{n} \rightarrow \infty(n \rightarrow \infty)$. Then the following conditions are equivalent:

(a) There is a universal entire function for the sequence $C_{\varphi_{n}}: H(\mathbb{C}) \rightarrow H(\mathbb{C})$ $(n \geq 1)$ that is bounded on $\Gamma$.

(b) For each continuous function $\varphi:[0,+\infty) \rightarrow(0,+\infty)$ that is integrable on $[1,+\infty)$, there is a $\left(C_{\varphi_{n}}\right)$-universal entire function $f$ such that

$$
\lim _{\substack{z \rightarrow \infty \\ z \in \Gamma}} \exp \left(\varphi(|z|)|z|^{3 / 2}\right) f(z)=0 .
$$

(c) There is an Arakelian subset $\Gamma_{0} \subset \mathbb{C}$ with $\Gamma_{0} \supset \Gamma$ satisfying the following property: for every $R>0$ there exists $n \in \mathbb{N}$ such that $\Gamma_{0} \cap B\left(b_{n}, R\left|a_{n}\right|\right)=\emptyset$.

Proof. To prove that (b) implies (a), take $\varphi(t):=\frac{1}{1+t^{4 / 3}}$. Then there is a $C_{\varphi_{n}}{ }^{-}$ universal entire function $f$ with $\lim _{\substack{z \rightarrow \infty \\ z \in \Gamma}} \exp \left(\frac{|z|^{3 / 2}}{1+|z|^{4 / 3}}\right) f(z)=0$. Hence $\lim _{\substack{z \rightarrow \infty \\ z \in \Gamma}} f(z)=$ 0 , showing the boundedness of $f$ on $\Gamma$.

Assume that (a) holds for some $f$. Since $f$ is bounded on $\Gamma$, a theorem by Danielyan and Schmieder [15] tells us that the set $\Gamma_{0}:=\{z \in \mathbb{C}:|f(z)| \leq$ 
$\left.\sup _{\Gamma}|f|\right\}$ is an Arakelian set. Obviously, $\Gamma_{0} \supset \Gamma$. Suppose that there is $R>0$ such that $\Gamma_{0} \cap B\left(b_{n}, R\left|a_{n}\right|\right) \neq \emptyset$ for all $n \in \mathbb{N}$. Then there exists a sequence $\left\{z_{n}\right\}_{n \geq 1} \subset B(0, R)$ such that $\left\{\varphi_{n}\left(z_{n}\right)\right\}_{n \geq 1} \subset \Gamma_{0}$. Let $g(z):=1+M \in H(\mathbb{C})$, where $M:=\sup _{\Gamma}|f|=\sup _{\Gamma_{0}}|f|$. Then no subsequence of $\left(C_{\varphi_{n}} f\right)$ can approximate $g$ on the compact set $K=\bar{B}(0, R)$, because $\sup _{K}\left|C_{\varphi_{n}} f-g\right| \geq\left|f\left(\varphi_{n}\left(z_{n}\right)\right)-(1+M)\right| \geq$ $1+M-\left|f\left(\varphi_{n}\left(z_{n}\right)\right)\right| \geq 1(n \in \mathbb{N})$. This contradiction shows that (c) is true.

Finally, we start from (c). To prove (b), we can assume without loss of generality that $\Gamma=\Gamma_{0}$, so $\Gamma$ is itself an Arakelian set. Fix a continuous function $\varphi:[0,+\infty) \rightarrow(0,+\infty)$ such that $\int_{1}^{+\infty} \varphi(t) d t<+\infty$. Then the function $\varepsilon(t):=\exp \left(-\varphi(t) t^{3 / 2}-t^{1 / 3}\right)(t \geq 0)$ is continuous, positive and satisfies

$$
\int_{1}^{+\infty} t^{-3 / 2} \log \frac{1}{\varepsilon(t)} d t<+\infty
$$

Let $\left\{P_{n}: n \in \mathbb{N}\right\}$ be a dense countable subset of $H(\mathbb{C})$, for instance, the set of all polynomial whose coefficients have rational real and imaginary parts. We can construct a sequence $\left(Q_{n}\right)$ whose members are in $\left\{P_{n}: n \in \mathbb{N}\right\}$ such that every function $P_{m}$ occurs infinitely many times in $\left(Q_{n}\right)$. We denote $B_{n}:=\bar{B}(0, n)$ $(n \geq 1)$.

By hypothesis, given $R>0$ there is $n \in \mathbb{N}$ with $\Gamma \cap \bar{B}\left(b_{n}, R\left|a_{n}\right|\right)=\emptyset$. Let us show that, in fact, there are infinitely many of such $n$ 's. Indeed, if it were not true, there would be some $R>0$ and some $n_{0} \in \mathbb{N}$ with $\Gamma \cap \bar{B}\left(b_{n}, R\left|a_{n}\right|\right) \neq \emptyset$ for all $n>n_{0}$. Set $\widetilde{R}:=R+\max \left\{\frac{\operatorname{dist}\left(b_{k}, \Gamma\right)}{\left|a_{k}\right|}: 1 \leq k \leq k_{0}\right\}$. Then it is plain that $\Gamma \cap B\left(b_{n}, \widetilde{R}\left|a_{n}\right|\right) \neq \emptyset$ for all $n \geq 1$, which is absurd.

Now, select $n_{1} \in \mathbb{N}$ with $\Gamma \cap \bar{B}\left(b_{n_{1}},\left|a_{n_{1}}\right|\right)=\emptyset$. Since $b_{n}$ and $b_{n} / a_{n}$ tend to $\infty$, one derives $\left|b_{n}\right|-2\left|a_{n}\right|=\left|b_{n}\right|\left(1-2\left|a_{n} / b_{n}\right|\right) \rightarrow \infty$, so $\left|b_{n}\right|-2\left|a_{n}\right|>\left|b_{n_{1}}\right|+\left|a_{n_{1}}\right|$ for $n \geq m$, say. Take $n_{2}>\max \left\{n_{1}, m\right\}$ such that $\Gamma \cap \bar{B}\left(b_{n_{2}}, 2\left|a_{n_{2}}\right|\right)=\emptyset$. Observe that we also obtain $\left|z-b_{n_{1}}\right| \geq|z|-\left|b_{n_{1}}\right| \geq\left|b_{n_{2}}\right|-\left|b_{n_{2}}-z\right|-\left|b_{n_{1}}\right| \geq\left|b_{n_{2}}\right|-2\left|a_{n_{2}}\right|-\left|b_{n_{1}}\right|>$ $\left|a_{n_{1}}\right|$ for all $z \in \bar{B}\left(b_{n_{2}}, 2\left|a_{n_{2}}\right|\right)$. Hence $\bar{B}\left(b_{n_{1}},\left|a_{n_{1}}\right|\right) \cap \bar{B}\left(b_{n_{2}}, 2\left|a_{n_{2}}\right|\right)=\emptyset$. With this procedure, we may determine by induction a sequence $\left\{n_{1}<n_{2}<\cdots\right\} \subset \mathbb{N}$ satisfying $\Gamma \cap J_{k}=\emptyset=J_{j} \cap J_{k}=\emptyset(k, j \geq 1, k \neq j)$, where $J_{k}:=\bar{B}\left(b_{n_{k}}, k\left|a_{n_{k}}\right|\right)$. Note also that the balls $J_{k}$ tend to $\infty$ as $k \rightarrow \infty$ in the sense that $\min \{|z|: z \in$ $\left.J_{k}\right\} \rightarrow+\infty$ (indeed, these minima are $\left|b_{n_{k}}\right|-k\left|a_{n_{k}}\right|$ and the $n_{k}$ 's were chosen by using $\left|b_{n}\right|-R\left|a_{n}\right| \rightarrow+\infty$ for all $R>0$ ). Hence the closed sets $\Gamma, J_{1}, J_{2}, \ldots$ are mutually disjoint, the $J_{k}$ 's are compact with connected complement, $\Gamma$ is an Arakelian set and the $J_{k}$ 's do not accumulate at any finite point. From this it is easy to see that $A:=\Gamma \cup \bigcup_{n=1}^{\infty} J_{n}$ is also an Arakelian set.

Consider the function $g: A \rightarrow \mathbb{C}$ given by

$$
g(z)=\left\{\begin{array}{cl}
0 & \text { if } z \in \Gamma \\
Q_{k}\left(\frac{z-b_{n_{k}}}{a_{n_{k}}}\right) & \text { if } z \in J_{k}(k \in \mathbb{N}),
\end{array}\right.
$$

which is clearly continuous on $A$ and holomorphic in $A^{0}$. By (8) and a tangential approximation theorem due to Arakelian [16, pp. 160-162], there exists an entire 
function $f$ such that

$$
|f(z)-g(z)|<\varepsilon(|z|) \quad(z \in A) .
$$

From (9) one obtains

$$
\left|\exp \left(\varphi(|z|)|z|^{3 / 2}\right) f(z)\right|<\exp \left(-|z|^{1 / 3}\right) \longrightarrow 0 \quad(z \rightarrow \infty ; z \in \Gamma) .
$$

It remains to prove that $f \in \mathcal{U}\left(\left(C_{\varphi_{n}}\right)\right)$. Fix a compact set $K \subset \mathbb{C}$, an $\varepsilon \in(0,1)$ and a polynomial $P_{m}$. Since the sets $J_{k}$ escape towards $\infty$, we can find $k_{0} \in \mathbb{N}$ such that, for all $k \geq k_{0}, K \subset B_{k}$ and $|z|>(\log (1 / \varepsilon))^{1 / 3}$ for all $z \in J_{k}$. Select $k \geq k_{0}$ with $Q_{k}=P_{m}$. From (9), we get $\left|f(z)-Q_{k}\left(\frac{z-b_{n_{k}}}{a_{n_{k}}}\right)\right|<\varepsilon\left(z \in J_{k}\right)$, or equivalently, $\left|\left(C_{\varphi_{n_{k}}} f\right)(z)-P_{m}(z)\right|<\varepsilon\left(z \in B_{k}\right)$. Since $K \subset B_{k}$, the function $C_{\varphi_{n_{k}}} f$ is in the basic neighborhood $U\left(P_{m}, \varepsilon, K\right)$. Thus, each $P_{m}$ belongs to the closure of $\left\{C_{\varphi_{n}} f\right\}_{n \geq 1}$. Finally, the density of $\left(P_{m}\right)$ in $H(\mathbb{C})$ shows the universality of $f$.

Remark 3.6. 1. By using the approach of $[6]$ it can be demonstrated the equivalence of (a), (b), (c) of Theorem 3.5 to the following property:

Given a continuous function $\varphi:[0,+\infty) \rightarrow(0,+\infty)$ that is integrable on $[1,+\infty)$, there is a dense linear subspace $M \subset H(\mathbb{C})$ such that any function $f \in M \backslash\{0\}$ is $\left(C_{\varphi_{n}}\right)$-universal and satisfies $\lim _{\substack{z \in \Gamma \\ z \in \Gamma}} \exp \left(\varphi(|z|)|z|^{3 / 2}\right) f(z)=0$.

2. If $\Gamma$ is a Carleman set (see definition in the proof of Theorem 3.3), $\left(\varphi_{n}\right)$ is as in Theorem 3.5 and, for every $R>0$, there is $n \in \mathbb{N}$ such that $\Gamma \cap \bar{B}\left(b_{n}, R\left|a_{n}\right|\right)=\emptyset$, then with essentially the same proof of Theorem 3.5 we obtain the following: given any continuous function $\omega: \mathbb{C} \rightarrow(0,+\infty)$, there is $f \in \mathcal{U}\left(\left(C_{\varphi_{n}}\right)\right)$ such that $\lim \underset{\substack{z \rightarrow \infty \\ z \in \Gamma}}{ } \omega(z) f(z)=0$. Just use Nersesjan's instead of Arakelian's theorem and observe that the set $A=\Gamma \cup \bigcup_{k=1}^{\infty} J_{k}$ constructed in the last proof is a Carleman set.

\section{Frequent universality}

As said in Section 1, Bayart and Grivaux [3] established the frequent hypercyclicity of every translation operator $\tau_{a}\left(a \in \mathbb{C}^{*}\right)$. By refining the approach of [3] we are going to prove that, under certain conditions, a more general sequence $\left(C_{\varphi_{n}}\right)$ with $\left(\varphi_{n}\right) \subset \operatorname{Aut}(\mathbb{C})$ can be frequent universal.

Theorem 4.1. Let $\left\{\varphi_{n}(z)=a_{n} z+b_{n}\right\}_{n \geq 1} \subset \operatorname{Aut}(\mathbb{C})$. Assume that there exists an unbounded nondecreasing sequence $\left\{\omega_{n}\right\}_{n \geq 1} \subset(0,+\infty)$ satisfying

(a) $\left|b_{n}\right|-\omega_{n}\left|a_{n}\right| \rightarrow+\infty$ as $n \rightarrow \infty$, and

(b) $\left|b_{m}-b_{n}\right| \geq \omega_{m-n}\left(\left|a_{m}\right|+\left|a_{n}\right|\right)$ for all $m, n \in \mathbb{N}$ with $m>n$.

Then $\left(C_{\varphi_{n}}\right)$ is frequently universal on $H(\mathbb{C})$. 
Proof. According to [3, Lemma 2.2], there exist pairwise disjoint subsets $A(l, \nu)$ $(l, \nu \geq 1)$ of $\mathbb{N}_{0}:=\mathbb{N} \cup\{0\}$ of positive lower density such that, for any $n \in A(l, \nu)$ and $m \in A(k, \mu)$, we have

$$
\begin{gathered}
n \geq \nu \quad \text { and } \\
|n-m| \geq \nu+\mu \text { if } n \neq m .
\end{gathered}
$$

Let $\left\{P_{l}\right\}_{l \geq 1}$ be a dense sequence in $H(\mathbb{C})$, and let $\left\{n_{k}\right\}_{k \geq 1}$ be the increasing sequence of elements of $\bigcup_{l, \nu \geq 1} A(l, \nu)$. If $n_{k} \in A(l, \nu)$ then we define $B_{k}$ as

$$
B_{k}=\varphi_{n_{k}}\left(\bar{B}\left(0, \omega_{\nu} / 2\right)\right)=\bar{B}\left(b_{n_{k}}, \omega_{\nu}\left|a_{n_{k}}\right| / 2\right) .
$$

Observe that the balls $B_{k}$ are pairwise disjoint. Indeed, if $n=n_{k} \in A(l, \nu)$ and $m=n_{k^{\prime}} \in A(j, \mu)$ with $k^{\prime}>k$, then $m>n$ and the distance between the centers of $B_{k}, B_{k^{\prime}}$ is $\left|b_{m}-b_{n}\right| \geq\left(\left|a_{m}\right|+\left|a_{n}\right|\right) \omega_{m-n} \geq\left(\left|a_{m}\right|+\left|a_{n}\right|\right) \omega_{\mu+\nu} \geq\left(\left|a_{m}\right|+\right.$ $\left.\left|a_{n}\right|\right)(1 / 2)\left(\omega_{\mu}+\omega_{\nu}\right)>(1 / 2) \omega_{\mu}\left|a_{m}\right|+(1 / 2) \omega_{\nu}\left|a_{n}\right|=\operatorname{radius}\left(B_{k}\right)+\operatorname{radius}\left(B_{k^{\prime}}\right)$, where (b), (11) and the fact that $\left(\omega_{n}\right)$ is nondecreasing have been used. Now, from (a), (10) and the fact that $\left(\omega_{n}\right)$ is nondecreasing we obtain

$$
\min _{z \in B_{k}}|z|=\left|b_{n_{k}}\right|-\frac{1}{2} \omega_{\nu}\left|a_{n_{k}}\right|>\left|b_{n_{k}}\right|-\omega_{\nu}\left|a_{n_{k}}\right| \geq\left|b_{n_{k}}\right|-\omega_{n_{k}}\left|a_{n_{k}}\right| \rightarrow+\infty(k \rightarrow \infty) .
$$

From the closedness and disjointness of the $B_{k}$ 's and the fact that they escape to $\infty$, it is easy to realize that the set $F:=\bigcup_{k=1}^{\infty} B_{k}$ is a Carleman set (see the proof of Theorem 3.3). Define the functions $\alpha: F \rightarrow \mathbb{C}$ and $g: F \rightarrow \mathbb{C}$ as $\alpha(z)=\frac{1}{1+|z|}$ $(z \in F)$ and $g(z)=P_{l}\left(\frac{z-a_{n_{k}}}{b_{n_{k}}}\right)\left(z \in B_{k}, n_{k} \in A(l, \nu), k \in \mathbb{N}\right)$. Then $\alpha$ and $g$ are continuous on $F, \alpha$ is positive and $\alpha \in H\left(F^{0}\right)$. According to Nersesjan's tangential approximation theorem, there is $f \in H(\mathbb{C})$ such that

$$
|f(z)-g(z)|<\alpha(z) \quad(z \in F) .
$$

We claim that $f$ is frequently universal for $\left(C_{\varphi_{n}}\right)$. To see this, fix $l, \nu \in \mathbb{N}$ and $\varepsilon>0$. By (a), we can choose $n_{0} \in \mathbb{N}$ such that $\left|b_{n}\right|-\omega_{n}\left|a_{n}\right|<1 / \varepsilon$ for every $n \geq n_{0}$. From (10) and since $\left(\omega_{n}\right)$ is nondecreasing we get for all $z \in \bar{B}\left(0, \omega_{\nu} / 2\right)$ that

$$
\left|\varphi_{n}(z)\right|=\left|b_{n}+a_{n} z\right| \geq\left|b_{n}\right|-|z|\left|a_{n}\right| \geq\left|b_{n}\right|-\frac{\omega_{\nu}}{2}\left|a_{n}\right| \geq\left|b_{n}\right|-\omega_{n}\left|a_{n}\right|>\frac{1}{\varepsilon} .
$$

Pick $k_{0} \in \mathbb{N}$ with $n_{k_{0}}>n_{0}$. Then for all $z \in \bar{B}\left(0, \omega_{\nu} / 2\right)$ and all $k>k_{0}$ with $n=n_{k} \in A(l, \nu)$ we obtain from (12) and (13) that

$$
\left|\left(C_{\varphi_{n}} f\right)(z)-P_{l}(z)\right|=\left|f\left(\varphi_{n}(z)\right)-g\left(\varphi_{n}(z)\right)\right|<\frac{1}{1+\left|\varphi_{n}(z)\right|}<\varepsilon .
$$

Since the lower density of $A(l, \nu) \backslash\left\{n_{1}, \ldots, n_{k_{0}}\right\}$ is positive and the sets $\{g \in H(\mathbb{C})$ : $\left.\sup _{|z| \leq \omega_{\nu} / 2}\left|g(z)-P_{l}(z)\right|<\varepsilon\right\} \quad(\varepsilon>0 ; l, \nu \in \mathbb{N})$ form a basis of the topology of $H(\mathbb{C})$ (notice that the property $\omega_{\nu} \rightarrow \infty$ is crucial here) it follows the frequent universality of our $f$. 
Corollary 4.2. If $\left(b_{n}\right) \subset \mathbb{C}$ is a sequence such that

$$
\lim _{k \rightarrow \infty} \inf _{n \in \mathbb{N}}\left|b_{n+k}-b_{n}\right|=+\infty
$$

then the sequence of translations $\left(\tau_{b_{n}}\right)$ is frequently universal on $H(\mathbb{C})$.

Proof. Given $M>0$ there is $k_{0} \in \mathbb{N}$ such that $\left|b_{n+k}-b_{n}\right|>M+\left|b_{1}\right|$ for all $n \in \mathbb{N}$ and all $k \geq k_{0}$. Letting $n=1$ and using the triangle inequality we get $\left|b_{k}\right|>M$ for all $k \geq k_{0}+1$. Setting $b_{0}:=0$ one obtains $\left|b_{n+k}-b_{n}\right|>M$ for all $n \in \mathbb{N}_{0}$ and all $k \geq k_{0}+1$, hence $\delta_{k}:=\inf _{\substack{n \in \mathbb{N}_{0} \\ j>k}}\left|b_{n+j}-b_{n}\right| \rightarrow+\infty$ as $k \rightarrow \infty$. In particular $\delta_{k}>0$ for $k \geq N$, say. Consider the sequence $c_{n}:=b_{N n}$. Then the sequence $\omega_{k}=(1 / 2) \inf _{\substack{n \in \mathbb{N}_{0} \\ j>k}}\left|c_{n+j}-c_{n}\right|$ is positive, nondecreasing and unbounded, and fulfills (a) and (b) of Theorem 4.1 (with $c_{n}$ instead of $b_{n}$ and $a_{n}=1$ ). Therefore $\left(\tau_{b_{N n}}\right)$ is frequently universal, so $\left(\tau_{b_{n}}\right)$ also is because $(N n)$ has positive density $1 / N$ in the sequence $\mathbb{N}$.

Remark 4.3. 1. By selecting $a_{n}=1, b_{n}=$ an and $\omega_{n}=|a| n / 2$, we get the already known frequent hypercyclicity of $\tau_{a}(a \neq 0)$. As a different instance of Theorem 4.1, the sequence $\left(C_{\varphi_{n}}\right)$ is frequently universal if $\varphi_{n}(z)=a_{n} z+b_{n}:=n^{\alpha} z+n^{\beta}$, where $\beta>0$ and $\beta \geq 1+\alpha$. Indeed, let $\gamma:=\beta-\alpha$ and consider the continuous function $\varphi:(1,+\infty) \rightarrow(0,+\infty)$ defined by $\varphi(t):=\frac{t^{\beta}-1}{t^{\alpha}(t-1)^{\gamma}}$. Then $\lim _{t \rightarrow+\infty} \varphi(t)=$ $1>0$ and $\lim _{t \rightarrow 1+} \varphi(t)=\left\{\begin{array}{cl}\beta & \text { if } \gamma=1 \\ +\infty & \text { if } \gamma>1,\end{array}\right.$ so $d:=\inf _{t>1} \varphi(t)>0$. Set $\delta:=$ $\min \{1 / 2, d / 2\}$ and take $\omega_{n}:=\delta \cdot n^{\gamma}$. Then $\left(\omega_{n}\right)$ is positive, nondecreasing and unbounded. Moreover, $\left|b_{n}\right|-\omega_{n}\left|a_{n}\right| \geq n^{\beta}-(1 / 2) n^{\alpha} n^{\gamma}=(1 / 2) n^{\beta} \rightarrow+\infty(n \rightarrow \infty)$, which yields the condition (a) of Theorem 4.1. Finally, if $m>n$, one has

$$
\frac{\left|b_{m}-b_{n}\right|}{\omega_{m-n}\left(\left|a_{m}\right|+\left|a_{n}\right|\right)}=\frac{m^{\beta}-n^{\beta}}{\delta\left(m^{\alpha}+n^{\alpha}\right)(m-n)^{\gamma}} \geq \frac{m^{\beta}-n^{\beta}}{2 \delta m^{\alpha}(m-n)^{\gamma}}=\frac{1}{2 \delta} \varphi\left(\frac{m}{n}\right) \geq \frac{d}{2 \delta} \geq 1,
$$

that gives $(\mathrm{b})$.

2. It would be interesting to characterize the sequences of automorphisms of $\mathbb{C}$ generating frequent universality, as well as to characterize the (or, at least, to give some criterion yielding) sequences $\left(\varphi_{n}\right) \subset \operatorname{Aut}(\mathbb{C})$ generating frequent $\mathcal{M}$ universality, that is, such that there exists $f \in H(\mathbb{C})$ with the following property: for every $g \in H\left(\mathbb{C}^{*}\right)$, every $\varepsilon>0$ and every $K \in \mathcal{M}\left(\mathbb{C}^{*}\right)$, the set $\{n \in \mathbb{N}$ : $\left.\left|f\left(\varphi_{n}(z)\right)-g(z)\right|<\varepsilon \forall z \in K\right\}$ has positive lower density. In particular, we ask the questions:

(i) If $0<\alpha<1$, is $\left(C_{z \mapsto z+n^{\alpha}}\right)$ frequently universal?

(ii) If $|a|>1$, is $\left(C_{z \mapsto a^{n} z}\right)$ frequently $\mathcal{M}$-universal?

\section{References}

[1] Y. Abe, Universal functions on complex special linear groups. Communications in difference equations. Proceedings of the Fourth International Conference on Difference Equations, Poznán, Poland, 1998 (S. Elaydi, G. Ladas, J. Popenda and J. Rakowski, eds.), Gordon and Breach, 2000, pp. 1-8. 
[2] Y. Abe and P. Zappa, Universal functions on complex general linear groups. J. Approx. Theory 100 (1999), 221-232.

[3] F. Bayart and S. Grivaux, Frequently hypercyclic operators. Trans. Amer. Math. Soc. 358 (2006), 5083-5117.

[4] F. Bayart and E. Matheron, Dynamics of Linear Operators. Cambridge Tracts in Mathematics, Cambridge University Press, 2009.

[5] L. Bernal-González and A. Bonilla, Universality of entire functions bounded on closed sets. J. Math. Anal. Appl. 315 (2006), 302-316.

[6] L. Bernal-González, M.C. Calderón-Moreno and W. Luh, Dense-lineability of sets of Birkhoff-universal functions with rapid decay. J. Math. Anal. Appl. 363 (2010), 327-335.

[7] L. Bernal-González and A. Montes-Rodríguez, Universal functions for composition operators. Complex Variables Theory Appl. 27 (1995), 47-56.

[8] L. Bernal-González and J.A. Prado-Tendero, $\mathcal{U}$-operators. J. Austral. Math. Soc. (Series A) 78 (2005), 59-89.

[9] G.D. Birkhoff, Démonstration d'un théorème élémentaire sur les fonctions entières. C. R. Acad. Sci. Paris 189 (1929), 473-475.

[10] A. Bonilla, "Counterexamples" to harmonic Liouville's theorem and harmonic functions with zero nontangential limits. Colloq. Math. 83 (2000), 155-160.

[11] A. Bonilla and K.G. Grosse-Erdmann, On a theorem of Godefroy and Shapiro. Integr. Equ. Oper. Theory 56 (2006), 151-162.

[12] A. Bonilla and K.G. Grosse-Erdmann, Frequently hypercyclic operators and vectors. Ergod. Th. Dynam. Sys. 27 (2007), 383-404.

[13] M.C. Calderón-Moreno, Universal functions with small derivatives and extremely fast growth. Analysis 22 (2002), 57-66.

[14] G. Costakis and M. Sambarino, Genericity of wild holomorphic functions and common hypercyclic vectors. Adv. Math. 182 (2004), 278-306.

[15] A.A. Danielyan and G. Schmieder, Topological properties of level sets of entire functions. Results Math. 33 (1998), 266-273.

[16] D. Gaier, Lectures on Complex Approximation. Birkhäuser, Boston, 1985.

[17] T.L. Gharibyan, W. Luh and M. Niess, Birkhoff functions that are bounded on prescribed sets. Arch. Math. 86 (2006), 261-267.

[18] K.G. Grosse-Erdmann, Universal families and hypercyclic operators. Bull. Amer. Math. Soc. N. S. 36 (1999), 345-381.

[19] K.G. Grosse-Erdmann and R. Mortini, Universal functions for composition operators with non-automorphic symbol. J. Anal. Math. 107 (2009), 355-376.

[20] K.G. Grosse-Erdmann and A. Peris, Linear Chaos. Springer, New York, 2011.

[21] A.S.B. Holland, Introduction to the theory of entire functions. Academic Press, New York, 1973.

[22] W. Luh, On universal functions. Colloq. Math. Soc. János Bolyai 19 (1976), 503-511.

[23] W. Luh, Entire functions with various universal properties. Complex Variables Theory Appl. 31 (1996), 87-96.

[24] A.A. Nersesjan, Carleman sets. Izv. Akad. Nauk Armyan. SSR Ser. Mat. 6 (1971), 465-471 (in Russian). 
[25] M. Niess, Universelle Funktionen mit zusätzlichen Eigenschaften. Dissertation an der Universität Trier, 2006.

[26] R. Tenthoff, Universelle holomorphe Funktionen mit vorgegebene Approximationswegen. Shaker Verlag, Aachen, 2000.

[27] A. Vogt, On bounded universal functions. Preprint, 2011.

[28] P. Zappa, On universal holomorphic functions. Bollettino U. M. I. (7) 2-A (1988), 345-352.

Luis Bernal-González

Departamento de Análisis Matemático

Facultad de Matemáticas, Apdo. 1160

Avda. Reina Mercedes, 41080 Sevilla, Spain

e-mail: lbernal@us.es 